\title{
The role of three-dimensional reconstruction in laparoscopic partial nephrectomy for complex renal tumors
}

Jipeng Wang ${ }^{\dagger}$, Youyi Lư ${ }^{\dagger}$ Gang Wu, Tianqi Wang, Yongqiang Wang, Hongwei Zhao, Zhongbao Zhou and Jitao $\mathrm{Wu}^{*}(\mathrm{D}$

\begin{abstract}
Background: To evaluate the role of three-dimensional (3D) reconstruction technique in renal function protection and ipsilateral parenchymal mass preserved after laparoscopic partial nephrectomy (LPN) in patients with complex renal tumor (R.E.N.A.L.score $\geq 8$ ).

Methods: A retrospective study enrolling 49 patients who suffered from RCC and underwent LPN at our center, from October 1, 2017, to October 31, 2018. Twenty-one patients (group A) underwent LPN with the 3D reconstruction technique before surgery, and the other 28 patients (group B) not. Preoperative and postoperative ipsilateral parenchymal mass volume and ipsilateral glomerular filtration rate (GFR) were analyzed 3-5 days prior and 3 months after PN. In order to compare the two groups, Mann-Whitney $U$ test and chi-square tests were performed. The main limitation of this technique is that the volume calculations are partly performed manually.

Results: All patients' median renal score was 10 with no difference between the two groups $(P=0.89)$, and the median tumor size of the two groups was $3.2 \mathrm{~cm}$ (group A) and $3.3 \mathrm{~cm}$ (group B) respectively $(P=0.14)$. In addition, the median warm ischemia time of the two groups was 21 min (group $A$ ) and 26 min (group $B)(P=0.003$ ). In group $A$ and group $B$, the rate of preserved global GFR was $88 \%$ and $86 \%(P=0.06)$, preserved ipsilateral GFR was $80 \%$ and $77 \%(P=0.01)$, and preserved ipsilateral parenchymal was $84 \%$ and $80 \%(P=0.03)$ separately.
\end{abstract}

Conclusion: 3D reconstruction technique was a beneficial method for more renal function and more preserved renal parenchymal mass volume after LPN.

Trial registration: Yantai Yuhuangding Hospital, YHD[2017]212. Registered 1 January 2017 (prospectively registered), http://www.ytyhdyy.com/nav/103.htm.

Keywords: Laparoscopic partial nephrectomy, Three-dimensional reconstruction, Renal function

\section{Background}

Partial nephrectomy (PN) was recommended for patients who suffered localized renal cell carcinoma (RCC), especially for cT1a tumors (according to the Union for International Cancer Control TNM staging system), on the account of preserving more nephron and improving the postoperative renal functional outcomes [1-5]. However, surgeons occasionally had to transform the $\mathrm{PN}$ into the radical

\footnotetext{
* Correspondence: 13625350255@163.com

${ }^{+}$Jipeng Wang and Youyi Lu contributed equally to this work.

Department of Urology, The Affiliated Yantai Yuhuangding Hospital of

Qingdao University, NO. 20 East Yuhuangding Road, Yantai 264000,

Shandong, China
}

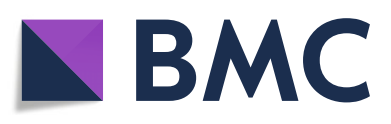

(c) The Author(s). 2019 Open Access This article is distributed under the terms of the Creative Commons Attribution 4.0 International License (http://creativecommons.org/licenses/by/4.0/), which permits unrestricted use, distribution, and

reproduction in any medium, provided you give appropriate credit to the original author(s) and the source, provide a link to the Creative Commons license, and indicate if changes were made. The Creative Commons Public Domain Dedication waiver (http://creativecommons.org/publicdomain/zero/1.0/) applies to the data made available in this article, unless otherwise stated. fore, the renal functional outcomes became uncertain. Additionally, a recent research indicated that $\mathrm{RN}$ can improve the probability of suffering chronic kidney disease (CKD) and decrease the survival rate [6]. So, it is crucial to preserve more nephron for the RCC patients in the early stage.

Normally, two-dimensional (2D) computed tomography $(\mathrm{CT})$ has occupied an important position in diagnosing the renal tumors [7]. However, traditional CT images were abstract and weak in describing anatomic structure for some complex tumors, such as renal hilar tumor and renal endophytic tumor. The actual position between tumor peripheral vessels was ambiguous, which 
increased the risk of additional damage in surgery. In recent years, one technology named three-dimensional (3D) CT reconstruction was applied to operation with the aim of decreasing the surgical accessory injury, maximizing the surgical accuracy, and protecting the postoperative organic function. In addition, this technique was comprehensively used in liver surgery [8], while seldom cases were reported in renal surgery.

In this study, we retrospectively analyzed renal functional outcomes after laparoscopic partial nephrectomy (LPN) as performed by an experienced surgeon, and compared preserved renal parenchymal volume after LPN by $3 \mathrm{D}$ reconstruction technique and traditional $2 \mathrm{D}$ CT scan separately.

\section{Methods}

\section{Patients}

The study protocol was approved by the Ethics Committee of the Affiliated Yantai Yuhuangding Hospital of Qingdao University (Yantai, Shandong), and informed consent was provided for the patients if they accepted the technique. From October 1, 2017, to October 31, 2018, 49 patients were required to take the abdominal CT scans with angiography and undergo LPN in our institution. Specifically for this research, since May 31, 2018, prior to the intervention, the eligible patients were told to take 3D reconstructive technique. So, the patients were divided into two groups (group A vs group B) in accordance with whether to take $3 \mathrm{D}$ reconstruction.

\section{Approach}

In our study, we evaluated the complexity of tumors by R.E.N.A.L. score [9]. All surgeries were performed by an experienced urologist (accomplished the LPN more than 300 procedures); besides, the choice of intraperitoneal or retroperitoneal route was decided by the surgeon's experience and patients' characteristics. For each patient, we collected the basic indices, such as age, sex, body mass index
(BMI), and Charlson comorbidity index (CCI); clinical indices, such as clinical tumor size, tumor growth pattern, and tumor's R.E.N.A.L. score; perioperative indices, such as operative time, ischemia time, and estimated blood loss (EBL); and postoperative indices, such as pathologic tumor stage and positive margins. All kinds of parameters were supposed to reflect patients' conditions adequately.

In addition, all enrolled patients were required to undergo examination for glomerular filtration rate (GFR) 3-5 days prior and 3 months after PN to evaluate the functional outcomes. The contrast-enhanced CT was supposed to be taken 3 months after PN to assess preserved parenchymal mass within the operated kidney. All operations of the two groups utilized the same kind of harmonic scalpel; besides, the dosage of the hemostatic agent was identical. Every case of our study utilized the "intraoperative doppler usg" during the operation, and the surgery only clamped the renal artery during the operation. Additionally, all of the volume (tumor and parenchymal mass) was calculated by cylindrical volume ratio method named Syngo Studio imaging software (Siemens, Washington, D.C.) [10], and the GFR was estimated by Modification-Diet-Renal-Disease-2 [11], and all patients were required to have nuclear renal scans (99mTc-mercaptoacetyltriglycine) to estimate the renal function within the operated kidney as the previous study described [12], because all 49 patients have two kidneys.

\section{Preparation for PN}

In group A, tumors were described in coronal plane, vertical plane, and the cross-section by the $3 \mathrm{D}$ reconstructive technique (IQQA; EDDA Technology, Princeton, NJ, USA). Compared with the traditional CT scans, the tumors' accurate location, peripheral vessels, and collective system were easily acquired $[13,14]$. The profile of tumor was vividly presented to the surgeon (Fig. 1 describes the difference between traditional $\mathrm{CT}$ scans and $3 \mathrm{D}$ reconstructive $\mathrm{CT}$ scans). In addition, the reconstructive image was similar to

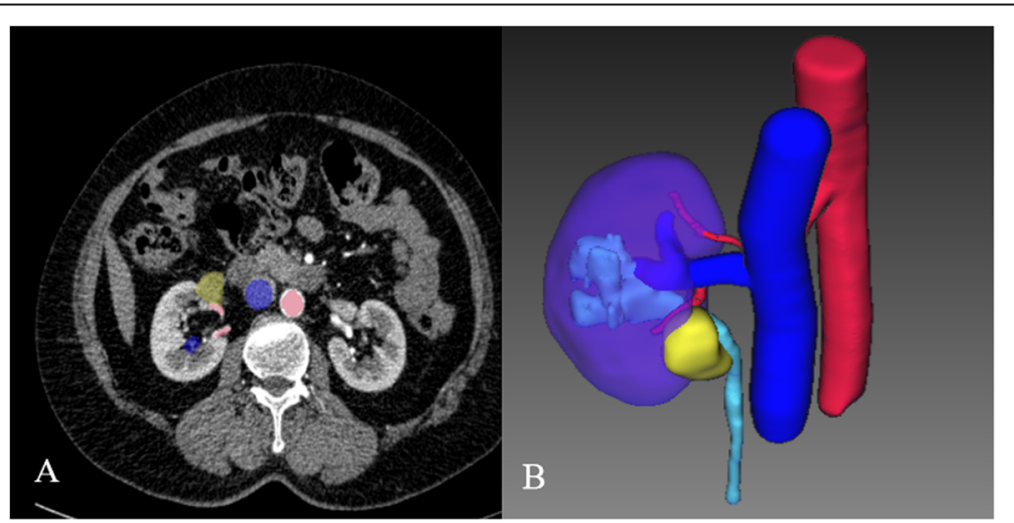

Fig. 1 Renal hilar tumor's 3D reconstruction and traditional CT scans. a General CT scans; red reflects artery, blue reflects vein, and yellow reflects tumor. $\mathbf{b}$ The reconstructive urinary system which include lesion, kidney, collective system, and blood vessels 
the real anatomic structure. The surgeon watched the reconstructive CT on a normal display and analyzed the disease before the operation, which helped the surgeon accomplish a clear impression for complex lesion.

When it came to traditional CT scans, the mass's diameters can only be measured by ordinary $2 \mathrm{D}$ images, and single dimensionality image presented an abstract impression for the surgeon. For some complex renal tumors, especially the endophytic tumor, the exact comparative location between the mass and vessels can only be detected by surgeon's experience, which occupied plenty of time for surgical processing, increasing the anesthesia risk. Figure 2 presented two kinds of completely endophytic renal tumor after being reconstructed. Additionally, tumors located in renal hilar may associate with some tiny, secluded vessels which were easily neglected and injured during operation. Hemorrhagic shock after surgery is likely to happen once surgeon neglected these small vessels injured.

\section{Statistical analysis}

We utilized the Statistical Package for the Social Sciences (SPSS) version 25.0 (IBM, Armonk, NY, USA) to carry out the data analysis. All of the continuous variables (e.g., warm ischemia time, operative time, clinical tumor size, GFR) were described by median and inter-quartile range (IQR). The significance between categorical variables such as sex, tumor growing patterns, and pathologic tumor stage was analyzed by chi-square test. Besides, the significance of continuous arguments was compared by using the Mann-Whitney $U$ test. Any differences between variable variate were significant if the $P<0.05$.

\section{Result}

\section{Patient characteristics}

All 49 patients accomplished undergoing the LPN with available data to study the global and ipsilateral GFR; operated renal parenchymal mass was preserved; besides, no patient died during the perioperative period. Group A included 21 patients who finally undertook the 3D reconstructive technique while other 28 patients were included in group B. In the two groups, patients' median age was 56 and 60 , respectively $(P=0.3)$, and the male's retention occupied $71 \%$ and $61 \%(P=0.4)$. All 49

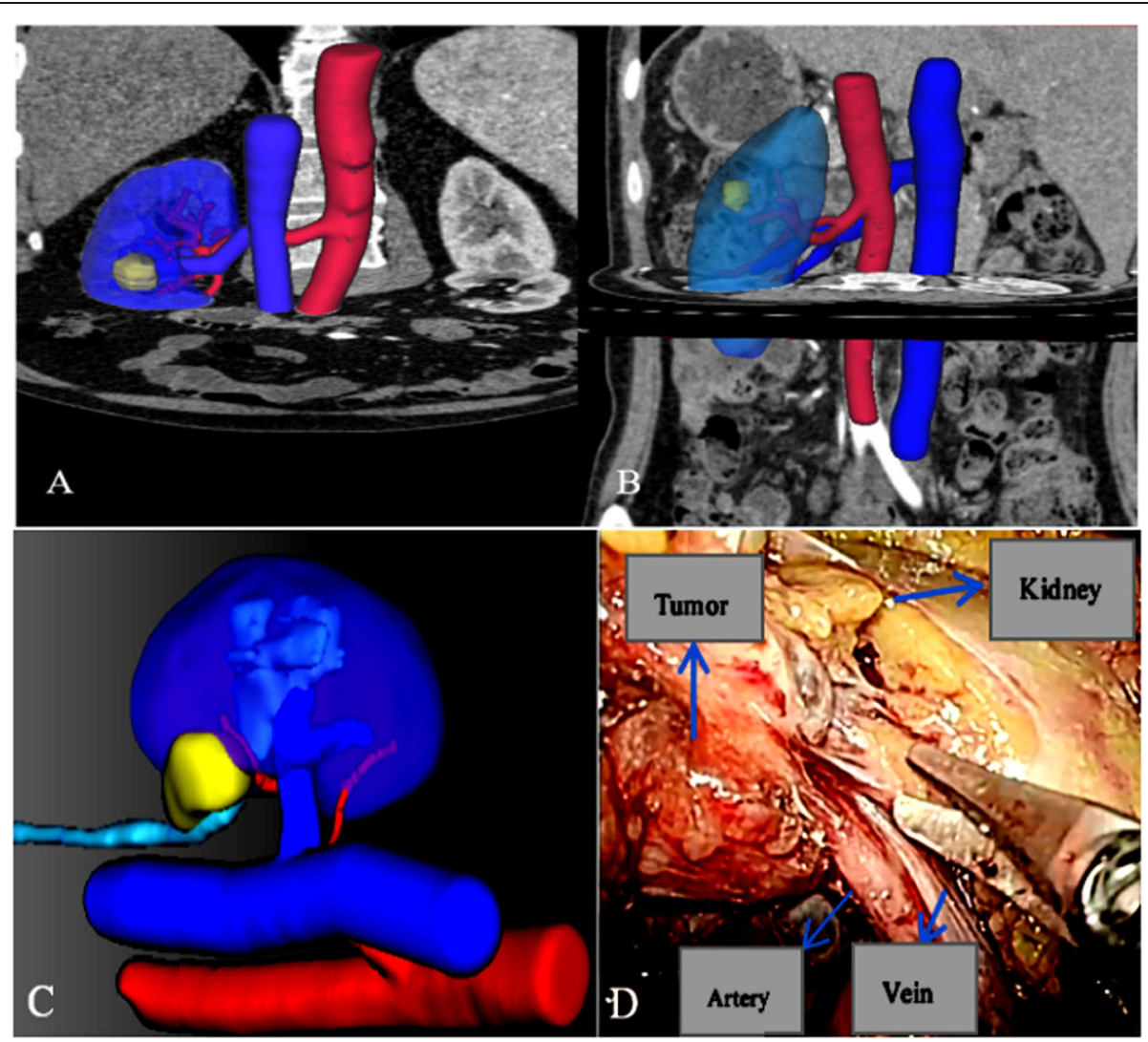

Fig. 2 Two cases of completely endophytic renal tumors; the reconstructed model and real operative visual. a A completely endophytic tumor was located near the renal hilar. b A completely endophytic renal tumor was located in the upper pole. c Renal hilar tumor's reconstruction, the tumor near a tiny renal artery. d Real operative visual showing the kidney, artery, and vein, and the relationship was alike with reconstructive image 
patients' median renal score was 10 (IQR 8-12 versus IQR $8-11, P=0.89$ ), and the median tumor size of patients in the two groups was $3.2 \mathrm{~cm}$ (IQR $2.1-4.3 \mathrm{~cm}$ ) and $3.3 \mathrm{~cm}$ (IQR $2.3-4.2 \mathrm{~cm}$ ), which showed no significance $(P=0.14)$. Additionally, renal hilar tumor and renal endophytic tumor were present in 15 and 6 patients in group A, while 15 and 13 patients in group B $(P=0.20)$, respectively, and the different distribution of renal hilar tumor between the two groups had no significance $(P=0.19)$, so as the endophytic tumor $(P=0.21)$. For operative time, $112 \mathrm{~min}$ (IQR 97-117 min) and 115 min (IQR 103-128 $\mathrm{min}$ ) were respectively presented in group A and group B $(P=0.06)$. With regard to patients' pathologic tumor stage, pT1a and pT1b were filed in 12 and 9 patients in group $\mathrm{A}$ while 21 and 7 patients in group B $(P=0.23)$. Patients who accepted this technique had significant decrease in estimated blood loss $(129 \mathrm{~mL}$, IQR $100-200 \mathrm{~mL}$ versus $160 \mathrm{~mL}$, IQR $120-240 \mathrm{~mL}, P=$ 0.04). Compared with group $B$, patients had a shorter warm ischemia time in group $\mathrm{A}$, and the median ischemia time was $21 \mathrm{~min}$ (IQR $16-25 \mathrm{~min}$ ) and $26 \mathrm{~min}$ (IQR 21-28 min) respectively $(P=0.003)$. The tumor section time ( $9 \mathrm{~min}$, IQR $6-13 \mathrm{~min}$ versus $13 \mathrm{~min}$, IQR $13-16$ $\min , P=0.002)$ and renal suturing time $(11 \mathrm{~min}, \mathrm{IQR} 8-$ $15 \mathrm{~min}$ versus $16 \mathrm{~min}$, IQR $11-18 \mathrm{~min}, P=0.001$ ) also had an obvious decrease. Table 1 described the detailed patients' characteristics data. Moreover, only one patient who suffered an endophytic tumor got a positive margin in group B.

\section{Functional outcomes}

For functional outcomes of the two groups, the median global preoperative GFR was $71 \mathrm{~mL} / \mathrm{min} / 1.73 \mathrm{~m}^{2}$ and 73 $\mathrm{mL} / \mathrm{min} / 1.73 \mathrm{~m}^{2}(P=0.57)$ while the postoperative global GFR was $66 \mathrm{~mL} / \mathrm{min} / 1.73 \mathrm{~m}^{2}$ and $64 \mathrm{~mL} / \mathrm{min} / 1.73 \mathrm{~m}^{2}$ $(P=0.85)$. Meanwhile, no significance was found in preserved global GFR (the median preserved rate of GFR was $88 \%$ and $86 \%$ respectively, $P=0.06$ ). The data of our letter inferred the organic compensation balanced out some functional regression for global functional outcomes. With regard to the operated kidney, the preoperative median ipsilateral GFR had no significance (44 $\mathrm{mL} / \mathrm{min} / 1.73 \mathrm{~m}^{2}$ versus $42 \mathrm{~mL} / \mathrm{min} / 1.73 \mathrm{~m}^{2}, \quad P=0.70$ ). However, the postoperative GFR of group A was higher than that of group B $\left(36 \mathrm{~mL} / \mathrm{min} / 1.73 \mathrm{~m}^{2}\right.$ versus $33 \mathrm{~mL} /$ $\mathrm{min} / 1.73 \mathrm{~m}^{2}, P=0.04$ ); besides, comparing with group $B$, patients in group A had a higher preserved GFR $(80 \%$ versus $77 \%, P=0.01$ ). For preoperative ipsilateral parenchymal mass, the median volume was present $168 \mathrm{~cm}^{3}$ and $171 \mathrm{~cm}^{3}$ in group A and group B respectively $(P=$ 0.61 ). Of notes, there was a significant difference in the postoperative ipsilateral parenchymal mass (median

Table 1 Patient characteristics

\begin{tabular}{|c|c|c|c|}
\hline Variable & Group A & Group B & $P$ \\
\hline No. of patients & 21 & 28 & \\
\hline Age (years), median (IQR) & $56(45-68)$ & $60(48-72)$ & 0.3 \\
\hline Male, $n(\%)$ & $15(71 \%)$ & $17(61 \%)$ & 0.4 \\
\hline BMI, kg/m, median (IQR) & $23(19-28)$ & $24(21-28)$ & 0.4 \\
\hline $\mathrm{CCl}$, median (IQR) & $2(1-3)$ & $1(0-2)$ & 0.6 \\
\hline Clinical tumor size (cm), median (IQR) & $3.2(2.1-4.3)$ & $3.3(2.3-4.2)$ & 0.14 \\
\hline R.E.N.A.L. score, median (IQR) & $10(8-12)$ & $10(8-11)$ & 0.89 \\
\hline Tumor growth pattern (\%) & & & 0.20 \\
\hline Renal hilar tumor & $15(71)$ & $15(53)$ & 0.19 \\
\hline Endophytic & $6(29)$ & $13(47)$ & 0.21 \\
\hline Warm ischemia time (min), median (IQR) & $21(16-25)$ & $26(21-28)$ & 0.003 \\
\hline Tumor resection time (min), median (IQR) & $9(6-13)$ & $13(10-16)$ & 0.002 \\
\hline Suturing time (min), median (IQR) & $11(8-15)$ & $16(11-18)$ & 0.001 \\
\hline $\mathrm{EBL}(\mathrm{mL})$, median (IQR) & $129(100-200)$ & $160(120-240)$ & 0.04 \\
\hline Operative time (min), median (IQR) & $112(97-117)$ & $115(103-128)$ & 0.06 \\
\hline Pathologic tumor stage (\%) & & & 0.23 \\
\hline pT1a & $12(73)$ & $21(65)$ & \\
\hline PT1b & $9(27)$ & $7(35)$ & \\
\hline Positive margins (\%) & $0(0)$ & $1(2)$ & 0.4 \\
\hline
\end{tabular}

BMI body mass index; CCI Charlson comorbidity index; IQR interquartile range; R.E.N.A.L. (R)adius (tumor size as maximal diameter), (E)xophytic/endophytic properties of tumor, (N)earness of tumor deepest portion to collecting system or sinus, (A)nterior (a)/posterior (p)descriptor and (L)ocation relative to polar lines; EBL estimated blood loss 
volume $157 \mathrm{~cm}^{3}$ versus $140 \mathrm{~cm}^{3}$, group A versus group $\mathrm{B}, P=0.02)$. The patients in group A had a higher percentage of preserved kidney (84\% versus $80 \%$, group A versus group $\mathrm{B}, P=0.03)$. The detailed functional outcomes were presented in Table 2.

\section{Discussion}

PN was recognized as the gold standard for dealing with localized renal tumor [1], because it had a lower risk for CKD and higher overall survival rate compared to RN verified by several multi-center retrospective researches $[6,15,16]$. In addition, the quantities of preserved nephron were reported to be a fatal element which influenced postoperative renal functional outcomes if the extended warm ischemia could be avoided [17-20]; therefore, methods to protect the nephron became crucial. With respect to some complex renal tumors (R.E.N.A.L.score $\geq 8$ ), especially entirely endophytic renal tumors and renal hilar tumors, additional normal parenchyma was always removed in order to get a complete surgical margin. Wu et al. did a retrospective analysis which indicated removing additional parenchymal mass increased the risk to suffer from CKD after PN and the non-renal cancer-related survival (NRCRS) would be reduced [6]. Besides, functional decline also occurred during parenchymal resection and reconstruction associated with tumor excision if lesions were surrounded by complex and secluded vessels. In this study, 3D reconstructive technique accurately reconstructed the anatomical structure and presented the tiny vessels and tissues around the tumor, which was proved to be a beneficial method to preserve more parenchymal mass and postoperative ipsilateral GFR.

According to the American Urological Association (AUA) guideline, patients were supposed to take the contrast-enhanced CT before surgery to notarize the location of the lesion. However, 2D CT scans were not the most sufficient preoperative preparation for some complex lesions. Cases were reported to suffer from severe complications in several kinds of medical center after PN (hemorrhagic shock or positive margin); all mentioned cases had the same characteristics that the tumor was almost endophytic or neared from renal artery branch. In addition, Porpiglia et al. had indicated high accuracy 3D reconstructive technique succeeded in selectively clamping the branches of renal artery during long-time robot-assisted partial nephrectomy (RAPN) for complex renal masses, maximally decreasing the renal damages caused by ischemia [14]. Moreover, 3D reconstructive technique was widely reported to be effectively used for preoperative evaluation and surgical planning in hepatic alveolar echinococcosis (HAE) [21]. Above all, 3D reconstructive technique had the huge potential to explore for PN.

In our research, 21 patients finally underwent the 3D reconstructive technique, both of the EBL and warm ischemia time had a significant decrease. The result of our study concluded that $3 \mathrm{D}$ reconstruction contributed to the tumor resection part and renal suturing part of the surgical procedure. Additionally, the median EBL of group A was only $129 \mathrm{~mL}$ with shorter warm ischemia time and more preserved ipsilateral parenchymal mass. Besides, none of the patients were reported to have positive margin in group A. When removing the tumor, a preciser location and volume helped the surgeon avoid damaging the normal tissue as little as possible, so that the renal parenchymal mass can be improved as much as possible. Additionally, some tiny peripheral vessels may be injured during the section of tumor removing or reconstructing the kidney, causing hemorrhage during operation and making the operative visual misty, which added the warm ischemia time. However, with the help

Table 2 Renal function and parenchymal mass preserved

\begin{tabular}{|c|c|c|c|}
\hline Variable & Group A & Group B & $P$ (value) \\
\hline No. of patients & 21 & 28 & \\
\hline \multicolumn{4}{|l|}{ Analysis of global function (median, IQR) } \\
\hline Preoperative global GFR, mL/min/1.73m² (median, IQR) & $71(58-95)$ & $73(62-93)$ & 0.57 \\
\hline Postoperative global GFR, mL/min/1.73m² (median, IQR) & $66(49-83)$ & $64(47-81)$ & 0.85 \\
\hline Global GFR preserved (\%) (median, IQR) & $88(84-95)$ & $86(81-90)$ & 0.06 \\
\hline \multicolumn{4}{|l|}{ Analysis focused on the operated kidney (median, IQR) } \\
\hline Preoperative ipsilateral GFR, mL/min/1.73m² (median, IQR) & $44(35-53)$ & $42(32-55)$ & 0.70 \\
\hline Postoperative ipsilateral GFR, mL/min/1.73m² (median, IQR) & $36(25-45)$ & $33(24-42)$ & 0.04 \\
\hline Ipsilateral GFR preserved (\%) (median, IQR) & $80(67-89)$ & $77(63-84)$ & 0.01 \\
\hline Preoperative ipsilateral parenchymal mass, $\mathrm{cm}^{3}$ (median, IQR) & $168(147-200)$ & $171(159-198)$ & 0.61 \\
\hline Postoperative ipsilateral parenchymal mass, $\mathrm{cm}^{3}$ (median, IQR) & $157(128-186)$ & $140(112-162)$ & 0.02 \\
\hline Ipsilateral parenchymal mass preserved (\%) (median, IQR) & $84(77-92)$ & $80(74-83)$ & 0.03 \\
\hline
\end{tabular}


of $3 \mathrm{D}$ reconstructive technique, the tiny vessels which are around the lesion or included in the tumor can be discovered before the operation, which helped the surgeon avoid injuring the tiny vessels when removing the tumor or reconstructing the kidney and shortened the warm ischemia time for hemostasis. Hence, both of the tumor resection part and renal suturing part had an obvious decrease. Besides, protecting more vessels during operation can reduce the devascularized parenchymal mass, which improved the preserved renal parenchymal mass [22]. Hence, our letter's data provided a powerful proof to indicate $3 \mathrm{D}$ reconstructive technique helped surgeon integrally removed the tumor with a minimal margin and a great postoperative renal function.

Clinical findings supported that preserved renal parenchymal mass has been becoming the most meaningful factor to influence function outcomes after PN compared with the warm ischemia time [22]. GFR and parenchymal mass were recognized to be two advantageous factors to estimate functional outcomes [23-25]. Previous data indicated volume loss after PN was derived from the parenchymal resection and reconstruction associated with tumor excision [26-29]. What can we conclude from this study is that higher postoperative ipsilateral preserved GFR and more preserved ipsilateral parenchymal mass of group A bring a reasonable expectation that $3 \mathrm{D}$ reconstructive technique avoid damaging extra parenchyma and protect tiny vessels from being injured during reconstruction associated with tumor excision. Figure $2 \mathrm{c}$ and $\mathrm{d}$ described two kinds of images in describing two patterns of renal hilar tumors and compared the reconstructed image with the real operative visual.

Recently, research challenged that the ischemia injury was the primary determinant of ultimate function after PN; besides, Volpe et al. and Thompson et al. pointed that patients who underwent PN had a higher risk of suffering renal function decline if the warm ischemia time was at least more than $25 \mathrm{~min}$ [17, 30]. Data of our study presented the ischemia time was $21 \mathrm{~min}$ and $26 \mathrm{~min}$, respectively, the shorter ischemia time of group A may not prove better functional outcomes after PN, but optimized the tumor excision and less surgical difficulty.

Limitations of this study included the nature of retrospective design and analysis, and our study was non-randomized. In addition, outcomes within one single medical center and the 49 patients in the study scale might produce degree of bias. The background differences cannot be controlled entirely, which might influence the functional outcomes, and a long-term and larger-scale study was supposed to be performed to analyze further functional outcomes of patients. The strength of our study is that all of the patients underwent LPN, and we can ignore the impact for surgical options, paying much attention to the postoperative functional outcomes. Moreover, this is the first contrastive analysis to focus on the preserved ipsilateral parenchymal mass and evaluate functional outcomes between $3 \mathrm{D}$ reconstructive and traditional CT scans after LPN.

\section{Conclusion}

In conclusion, 3D reconstructive technique helps surgery get more comprehensive information before operation. In addition, more preserved renal parenchymal mass and shorter warm ischemia time contributed to achieve great renal function, which indicted that technique is worth to generalize in LPN. Moreover, more advanced technique is supposed to be applied for the operation and optimizing the functional outcomes after LPN; more lager sample size studies are required to validate our results.

\section{Abbreviations \\ 2D: Two-dimensional; 3D: Three-dimensional; AUA: American Urological Association; BMl: Body mass index; CCl: Charlson comorbidity index; CKD: Chronic kidney disease; CT: Computed tomography; EBL: Estimated blood loss; GFR: Glomerular filtration rate; IQR: Interquartile range; LPN: Laparoscopic partial nephrectomy; NRCRS: Non-renal cancer-related survival; PN: Laparoscopic partial nephrectomy; R.E.N.A.L.: (R)adius (tumor size as maximal diameter), (E)xophytic/endophytic properties of tumor, (N)earness of tumor deepest portion to collecting system or sinus, (A)nterior (a)/ posterior (p)descriptor and (L)ocation relative to polar lines; RAPN: Robot- assisted partial nephrectomy; RCC: Renal cell carcinoma; RN: Radical nephrectomy; SPSS: Statistical Package for the Social Sciences}

\section{Acknowledgements}

Not applicable.

\section{Authors' contributions}

JW and YL analyzed the data and wrote this manuscript. GW, TW and ZZ paid attention to collecting the diverse clinic data. JW designed the study project. YW and $\mathrm{HZ}$ contributed to the statistical analysis. All authors read and approved the final manuscript.

\section{Funding}

The study was funded by grants from the National Nature Science Foundation of China (Nos. 81572835; 81870525), Key Research and Development Program of Shandong Province (No. 2018GSF118118), The Projects of medical and health technology development program of Shandong province (2016WS0712).

Availability of data and materials

All data generated or analyzed during this study are included in this published article.

\section{Ethics approval and consent to participate}

The study protocol was approved by the Ethics Committee of the Affiliated Yantai Yuhuangding Hospital of Qingdao University (Yantai, Shandong).

Every patient who underwent the $3 \mathrm{D}$ reconstruction signed the informed consent form.

\section{Consent for publication}

Not applicable.

\section{Competing interests}

The authors declare that they have no competing interests.

Received: 12 June 2019 Accepted: 29 August 2019

Published online: 11 September 2019

\section{References}

1. Campbell S, Uzzo RG, Allaf ME, et al. Renal mass and localized renal cancer: AUA guideline. J Urol. 2017;198:520-9. 
2. Huang WC, Levey AS, Serio AM, et al. Chronic kidney disease after nephrectomy in patient with renal cortical tumours: a retrospective cohort study. Lancet Oncol. 2006;7:735-40.

3. Scosyrev E, Messing EM, Sylvester $R$, et al. Renal function after nephron sparing surgery versus radical nephrectomy: results from EORTC randomized trial. Eur Urol. 2014:65:372-7.

4. Pignot $G$, Bigot $P$, Bernhard JC. Nephron-sparing surgery is superior to radical nephrectomy in preserving renal function benefit even when expanding indications beyond the traditional 4-cm cutoff. Urol Oncol. 2014;32:1024-30.

5. Weight CJ, Larson BT, Fergany AF, et al. Nephrectomy induced chronic renal insufficiency is associated with increased risk of cardiovascular death and death from any cause in patients with localized cT1b renal masses. The J Urol. 2010:(4):1317-23.

6. Wu J, Suk-Ouichai C, Dong W, et al. Analysis of survival for patients with chronic kidney disease primarily related to renal cancer surgery. BJU Int. 2018;121:93-100.

7. Lim DJ, Carter MF. Computerized tomography in the preoperative staging for pulmonary metastases in patients with renal cell carcinoma. J Urol. 1993;(4):1112-4.

8. Radtke A, Nadalin S, Sotiropoulos G, et al. Computer-assisted operative planning in adult living donor liver transplantation: a new way to resolve the dilemma of the middle hepatic vein. World J Surg. 2007;31:175-85.

9. Alexander K, Robert G. The R.E.N.A.L nephrometry score: a comprehensive standardized system for quantitating renal tumor size, location and depth. J Urol. 2009;3:844-53.

10. Simmons MN, Fergany AF, Campbell SC. Effect of parenchymal volume preservation on kidney function after partial nephrectomy. J Urol. 2011;(2):405-10.

11. Levey AS, Bosch JP, Lewis JB, et al. A more accurate method to estimate glomerular filtration rate from serum creatinine: a new prediction equation. Modification of Diet in Renal Disease Study Group. Ann Intern Med. 1999;130:461-70

12. Dong W, Wu JT, Suk-Ouichai $C$, et al. Ischemia and functional recovery from partial nephrectomy: refined perspectives. Eur Urol focus. 2018;4:572-8.

13. Ukimura O, Nakamoto M, Gill IS. Three-dimensional reconstruction of renovascular-tumor anatomy to facilitate zero-ischemia partial nephrectomy. Eur Urol. 2012;(1):211-7.

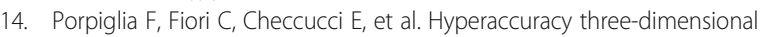
reconstruction is able to maximize the efficacy of selective clamping during robot-assisted partial nephrectomy for complex renal masses. Eur Urol. 2018;(5):651-60.

15. Choi SK, Song C. Risk of chronic kidney disease after nephrectomy for renal cell carcinoma. KJU. 2014;(10):636-42

16. Leppert JC, Lamberts RW, Thomas IC, et al. Incident CKD after radical or partial nephrectomy. J Am Soc Nephrol. 2017;1:207-16.

17. Volpe A, Blute ML, Ficarra $V$, et al. Renal ischemia and function after partial nephrectomy: a collaborative review of the literature. Eur Urol. 2015;68:61-74

18. Mir MC, Ercole C, Takagi T, et al. Decline in renal function after partial nephrectomy: etiology and prevention. J Urol. 2015;193:1889-98.

19. Simmons $\mathrm{MN}$, Hillyer $\mathrm{SP}$, Lee $\mathrm{BH}$, et al. Functional recovery after partial nephrectomy: effects of volume loss and ischemic injury. J Urol. 2012;187:1667-73.

20. Mir MC, Campbell RA, Sharma N, et al. Parenchymal volume preservation and ischemia during partial nephrectomy: functional and volumetric analysis. Urology. 2013;82:263-8.

21. He YB, Bai L, Aji T, et al. Application of $3 D$ reconstruction for surgical treatment of hepatic alveolar echinococcosis. World J Gastroenterol. 2015;(35):10200-7.

22. Dong W, Wu J, Suk-Ouichai C, et al. Devascularized parenchymal mass associated with partial nephrectomy: predictive factors and impact on functional recovery. J Urol. 2017:4:787-94.

23. Marconi L, Desai MM, Ficarra $V$, et al. Renal preservation and partial nephrectomy: patient and surgical factors. Eur Urol Focus. 2017;6:589-600.

24. Demirjian S, Lane BR, Derweesh $\mathrm{H}$, et al. Chronic kidney disease due to surgical removal of nephrons: relative rates of progression and survival. J Urol. 2014;192:1057-62.

25. Scosyrev $E$, Messing EM, Sylvester $R$, et al. Renal function after nephron-sparing surgery versus radical nephrectomy: results from EORTC randomized trial 30904. Eur Urol. 2014;65:372-7.

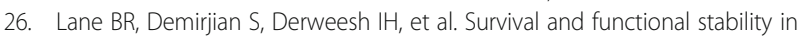
chronic kidney disease due to surgical removal of nephrons: importance of the new baseline glomerular filtration rate. Eur Urol. 2015;68:996-1003.

27. Choi JD, Park JW, Choi JY, et al. Renal damage caused by warm ischaemia during laparoscopic and robot-assisted partial nephrectomy: an assessment using Tc 99 m-DTPA glomerular filtration rate. Eur Urol. 2010;58:900-5.
28. Zargar $\mathrm{H}$, Akca O, Autorino $\mathrm{R}$, et al. Ipsilateral renal function preservation after robot assisted partial nephrectomy (RAPN): an objective analysis using mercapto-acetyltriglycine (MAG3) renal scan data and volumetric assessment. BJU Int. 2015:115:787-95.

29. Maurice MJ, Ramirez D, Malkoç E, et al. Predictors of excisional volume loss in partial nephrectomy: is there still room for improvement? Eur Urol. 2016;70:413-5.

30. Thompson RH, Lane BR, Lohse CM, et al. Every minute counts when the renal hilum is clamped during partial nephrectomy. Eur Urol. 2010;58:340-5.

\section{Publisher's Note}

Springer Nature remains neutral with regard to jurisdictional claims in published maps and institutional affiliations.
Ready to submit your research? Choose BMC and benefit from:

- fast, convenient online submission

- thorough peer review by experienced researchers in your field

- rapid publication on acceptance

- support for research data, including large and complex data types

- gold Open Access which fosters wider collaboration and increased citations

- maximum visibility for your research: over $100 \mathrm{M}$ website views per year

At $\mathrm{BMC}$, research is always in progress.

Learn more biomedcentral.com/submissions 